\title{
S5 Table
}

Distribution parameters of the HSMM (acceleration+angles) model for each state

\begin{tabular}{|l|l|l|l|l|l|l|l|l|l|}
\hline \multicolumn{7}{|l}{ acceleration } & \multicolumn{2}{l}{ anglex } & \multicolumn{2}{l}{ angley } & \multicolumn{2}{l}{ anglez } & duration \\
\hline state & mean & sigma & mean & sigma & mean & sigma & mean & sigma & lambda \\
\hline A & 0.000 & $5.5 \mathrm{E}-07$ & 5 & 0.0002 & 7 & 0.0001 & -48 & -0.0001 & $584 \mathrm{sec}$ \\
\hline B & 0.001 & $2.7 \mathrm{E}-06$ & 29 & 0.0006 & -11 & -0.0059 & 39 & -0.0045 & $393 \mathrm{sec}$ \\
\hline C & 0.007 & $6.3 \mathrm{E}-05$ & -35 & -0.0097 & 45 & -0.0083 & 8 & -0.0027 & $77 \mathrm{sec}$ \\
\hline D & 0.008 & $5.8 \mathrm{E}-05$ & 19 & 0.0025 & 16 & -0.0191 & -37 & -0.0023 & $86 \mathrm{sec}$ \\
\hline E & 0.008 & $1.3 \mathrm{E}-04$ & -51 & -0.0058 & -2 & 0.0046 & 35 & -0.0103 & $69 \mathrm{sec}$ \\
\hline F & 0.010 & $1.3 \mathrm{E}-04$ & -51 & 0.0147 & 4 & -0.0018 & -34 & -0.013 & $75 \mathrm{sec}$ \\
\hline G & 0.045 & $2.4 \mathrm{E}-03$ & 21 & -0.1807 & -2 & -0.0191 & 33 & -0.2734 & $60 \mathrm{sec}$ \\
\hline H & 0.056 & $1.7 \mathrm{E}-03$ & -37 & -0.0685 & 13 & 0.0055 & -13 & 0.0509 & $16 \mathrm{sec}$ \\
\hline I & 0.060 & $4.4 \mathrm{E}-03$ & -39 & 0.0099 & -40 & 0.0355 & -3 & 0.1478 & $87 \mathrm{sec}$ \\
\hline J & 0.309 & $1.2 \mathrm{E}-01$ & -28 & -0.0301 & -3 & -1.83 & 2 & 0.2618 & $20 \mathrm{sec}$ \\
\hline
\end{tabular}

